\title{
Service Scenarios for Green House Gas Monitoring Service over NGN
}

\author{
Sam-Gil Choi, Dong-Il Kim, and Soong-Hee Lee, Member, KIMICS
}

\begin{abstract}
Considerations for green house gas (GHG) monitoring over next generation network (NGN) are regarded as a green convergence service for the successful reduction of GHG emission leading to resolve global warming issue in that NGN is expected to provide secure connections to fixed-and-mobile converged (FMC) features. Model-based scenario approach is an appropriate way to standardize and actualize the desired service. This paper first describes the service scenario of GHG monitoring service over NGN.
\end{abstract}

Index Terms - Green House Gas, GHG Monitoring, Next Generation Network

\section{INTRODUCTION}

GHG monitoring is regarded as all activities for accounting and reporting of the direct and indirect GHG emission defined in GHG protocol[1]. Traditionally, GHG measurements were performed on the ground and collected on the limited domains. Even the advanced GHG inventory running on Web-based Internet may be vulnerable to the external threats or hackings. NGN looks the proper choice to satisfy these requirements[2]. In addition, ITU-T, responsible for the standardization of NGN, has been active on the efforts of ICT for preventing climate changes.

This paper first describes the service model for GHG monitoring over NGN. Then service scenarios for GHG monitoring over NGN are described and conclusions are lastly given.

\section{GHG MONITORING OVER NGN}

GHG monitoring is based on the existence of GHG inventory to expose the GHG sources originated from the corporate activities and systematically manage the amount of GHG emission. In addition, the safe transaction of the information on GHG inventory, that should be maintained for clean development mechanism (CDM) related businesses, leads to the service deployment over NGN with secured connections and FMC features.

\footnotetext{
Manuscript received July 7, 2011; revised August 1, 2011; accepted August 10, 2011

Sam-gil Choi is with the department of Information Communication Engineering, Dong-eui University (sgchoi@deu.ac.kr)
}

GHG monitoring over NGN may require the categorized and standardized procedures and functions for more efficient and meaningful results from the dispersed data worldwide. Hence, it is important to actualize the service scenario for the deployment of GHG monitoring service over NGN. Figure 1 shows an expected network configuration for GHG monitoring that consists of GHG sensor network (GSN), NGN and other components.

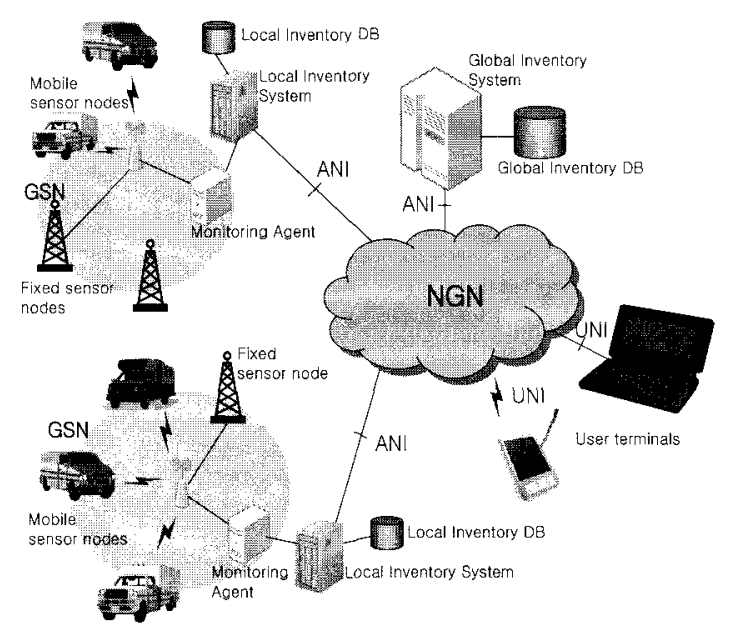

Fig. 1. Expected network configuration for the GHG monitoring service over NGN.

There may exist service features necessary for this service as follows:

- Managing the GHG inventories;

- Managing organizational boundaries;

- Providing sufficient data to the GHG inventory for decision making;

- Tracking the GHG emissions over time;

- Identifying and calculating the GHG emissions;

- Trading emission allowances among organizations, companies, or countries;

- GHG reporting and accounting;

- Maintaining the GHG monitoring infrastructure (which may include managing the inventory quality);

- etc. 


\section{SERVICE SCENARIO FOR GHG}

\subsection{GHG inventory managing}

GHG inventory maintains collected data and analyzed information in the GHG monitoring service. The inventory is managed by the authorized operators, who are also users of this service feature. The users can manage the GHG local or global inventory in the remote side through NGN. User terminals can be used to request or manage the service.

GHG inventory managing consists of the following procedures: requesting the GHG inventory managing service from the persons, organizations or companies; Authentication, Authorization, and Accounting (AAA) processes for the request; preparing the required information base according to the requested inventory framework; updating the GHG inventory framework; adding the missed or new items into the inventory; deleting the unused items in the inventory.

Flow for the GHG inventory managing at the global/local inventory is given in the figure 2 .

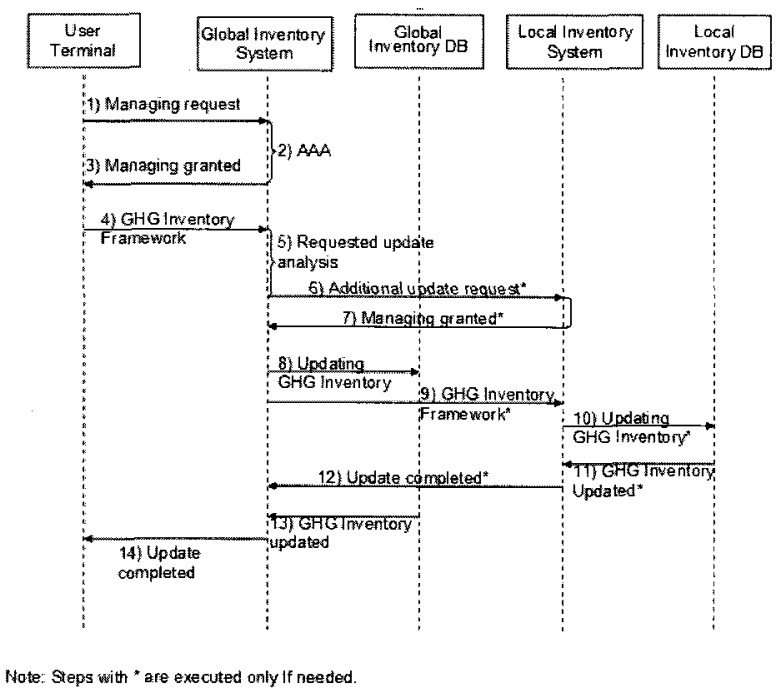

Fig. 2. Flow for the GHG Inventory Managing at the global inventory.

The Global Inventory System carries out the AAA procedure for the requesting User and User Terminal. If the AAA result is valid then the Global Inventory System grants the GHG Inventory Managing to the requesting User Terminal. Otherwise, it notifies the denial of the managing to the User Terminal (via ANI/UNI). The granted User Terminal sends the prepared GHG Inventory Framework information to the Global Inventory System (via UNI/ANI). The Global Inventory System analyzes the received GHG Inventory Framework information for deciding whether additional updates are necessary at the Local Inventory System. If additional updates are needed, the Global Inventory System sends additional update request to the Local Inventory System $\left(\right.$ via $\left.\mathrm{I}_{3}\right)$. Then the Local Inventory System grants the additional update request by notifying to the Global Inventory System (via $\mathrm{I}_{3}$ ). The Global Inventory System updates the GHG global inventory applying the received framework information. When updates at the global inventory are completed, the Global Inventory System sends the prepared GHG Inventory Framework information to the Local Inventory System (via $\mathrm{I}_{3}$ ). The Local Inventory System updates the GHG local inventory applying the received framework information. The Local Inventory System responses to the Global Inventory System for the update completed (via $\mathrm{I}_{3}$ ). The Global Inventory System finally responses to the User Terminal for the update completed (via ANI/UNI).

Another flow for the GHG Inventory Managing at the Local Inventory is given in the figure 3.

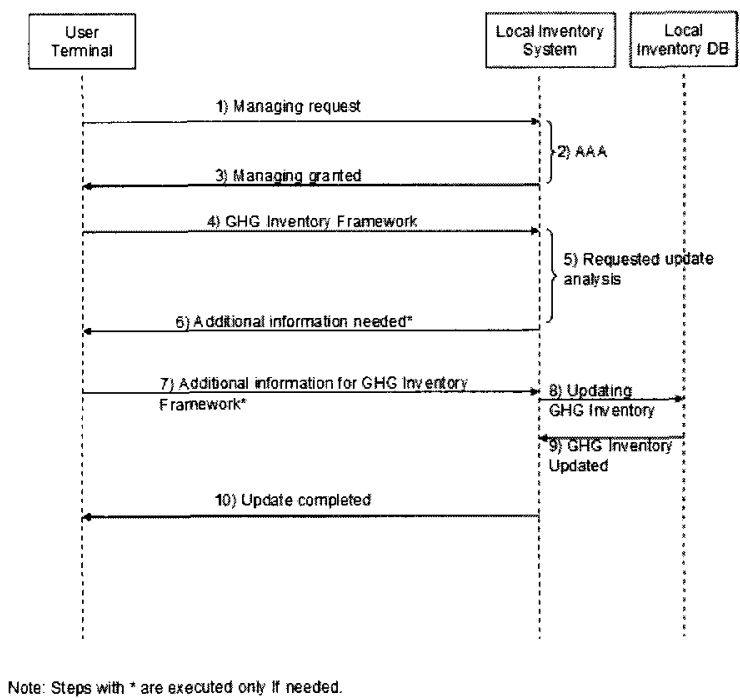

Fig. 3. Flow for the GHG Inventory Managing at the local inventory.

The User Terminal requests the GHG Inventory Managing to the Local Inventory System (via UNI/ANI). The Local Inventory System carries out the AAA procedure for the requesting User and User Terminal. If the AAA result is valid then the Local Inventory System grants the GHG Inventory Managing to the requesting User Terminal. Otherwise, it notifies the denial of the managing to the User Terminal (via ANI/UNI). The User Terminal sends the prepared GHG Inventory Framework information to the Local Inventory System (via UNI/ANI). The Local Inventory System analyzes the received GHG Inventory Framework information for deciding whether additional information is necessary at the Local Inventory System. The Local Inventory System notifies to the User when additional information is needed (via ANI/UNI). The User Terminal sends additional information for GHG Inventory framework to the Local Inventory System (via UNI/ANI). The Local Inventory System updates the GHG local inventory applying the received framework information and responses to the Global Inventory System for the update completed (via ANI/UNI). 


\subsection{GHG reporting}

The GHG reduction amount needs to be reported to the users periodically (e.g., annually, monthly, or weekly), or on user demands. The users can check the GHG reduction amount from the reports periodically or on demand in the local domain or global domain in any location if they can connect to NGN.

GHG reporting consists of the following procedures: requesting the GHG reporting from the persons, organizations or companies to the GHG monitoring service providers covering the periodic and on-demand basis; AAA processes for the requesting users or terminals; gathering the required information for guessing the GHG reduction amounts; deciding the GHG reduction amounts from the gathered information; transferring the GHG reporting information to the users or terminals. The information flows for each case are described in the figure 4 and 5.

The User Terminal requests the periodic GHG reporting to the Global/Local Inventory System (via UNI and ANI). The request includes the duration (reporting period, e.g., year, month, day, etc.) between each reporting. Then the Global/Local Inventory System carries out the AAA procedure for the requesting User and User Terminal. If the AAA result is valid then the Global/Local Inventory System grants the periodic GHG reporting to the requesting User Terminals and starts the timer for tracing the report period. Otherwise, it notifies the denial of the service to the User Terminal (via ANI and UNI). When the timer expires, the Global/Local Inventory System starts the actions for the periodic reporting by checking the availability GHG information for guessing the GHG reduction amounts. The Global Inventory System gathers GHG monitoring information from Global Inventory DB for guessing the GHG reduction amounts.

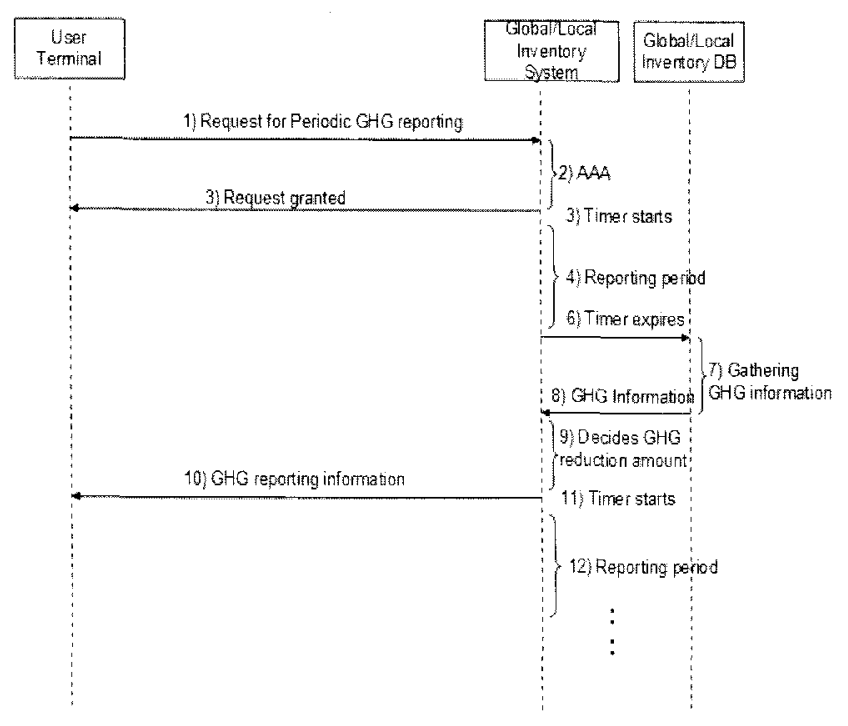

Fig. 4. Flow for the periodic GHG reporting.
It manipulates the GHG monitoring information for guessing GHG reduction amounts, decides the GHG reduction amounts for sending information to the requesting users, sends the decided GHG reporting information to the requesting terminal, and starts the timer again. The Global Inventory System repeats the upper processes when the timer expires.

The User Terminal requests the GHG reporting to the Global/Local Inventory System (via UNI and ANI). The Global/Local Inventory System carries out the AAA procedure for the requesting User and User Terminal. If the AAA result is valid then the Global/Local Inventory System grants the GHG reporting in the remote side to the requesting User Terminals and checks the availability of GHG information in the Global/Local Inventory DB. It checks the availability of GHG information in the Global/Local Inventory DB. GHG monitoring information is gathered from Global Inventory DB for guessing the GHG reduction amounts. Then Global Inventory System manipulates the GHG monitoring information for guessing GHG reduction amounts. It decides the GHG reduction amounts for sending information to the requesting users and sends the decided GHG reporting information to the requesting terminal.

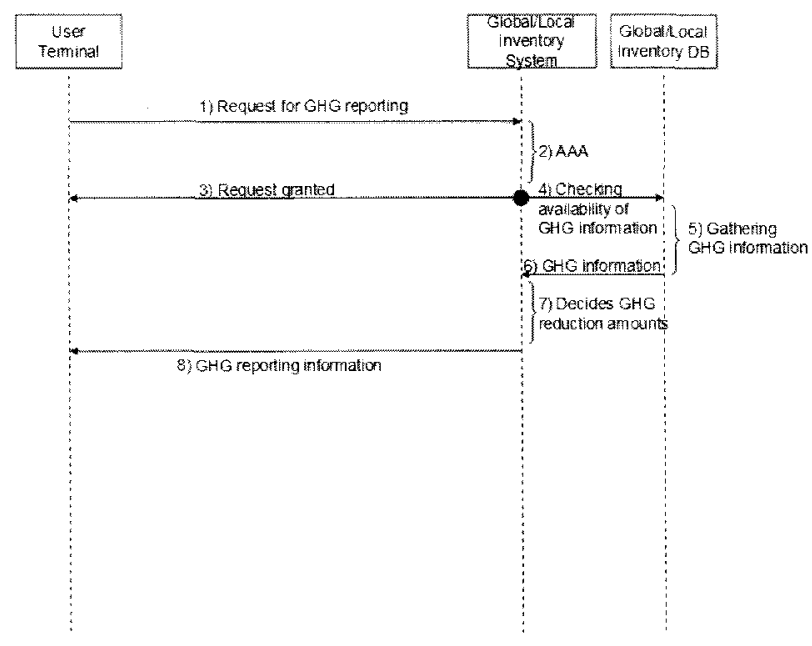

Fig. 5. Flow for the GHG reporting on demand.

\section{CONCLUSION AND FUTURE WORKS}

Service scenarios for GHG monitoring service over NGN are introduced in this paper. The proposed scenarios are expected to contribute as the framework or guideline to the future studies for standardization and actual service deployments. We focused on the service deployment issues applying the service scenario approach. After more enhancements, the proposed service model and scenarios will be verified in the future. Detailed procedures for data manipulation and calculation mechanisms are not considered here as they 
are mainly considered in the environmental engineering fields. Establishment and management of organizational, regional, and national boundaries are expected to be explored in the future study.

\section{ACKNOWLEDGMENT}

This work was supported by Dong-eui University Grant (2010AA169)

\section{REFERENCES}

[1] World Resources Institute(WRI), "Green house gas protocol: A Corporate Accounting and Reporting Standard", WRI, 2004.

[2] ITU-T Y.Sup.3: ITU-T Y.2000 series - Supplement on service scenarios for convergence services in a multiple network and application service provider environment", ITU-T Y.2000 series Y.Sup.3, ITU-T (2007)

[3] Young-gil Kim: Data transmission in u-ID sensor network configuration using Bluetooth, ICKIMICS 2004 Proceedings, KIMICS (2004)

[4] Sang-chul Shin, et. al.: Strategy for international standardization and wide spread of RFID/USN, KICS Journal, KICS (2004)

[5] IETF RFC 3761: The E.164 to Uniform Resource Identifiers (URI) Dynamic Delegation Discovery System (DDDS) Application (ENUM), IETF RFC 3761, IETF (2004)

[6] IETF RFC 4179: Using Universal Content Identifier (UCI) as Uniform Resource Names (URN), IETF RFC 4179, IETF (2005)

[7] ITU-T Y.IdMsec: NGN IDENTITY MANAGEMENT SECURITY, ITU-T Y.IdMsec, ITU-T (2007)

[8] ITU-T Y.2011, "General principles and general reference model for Next Generation Networks", ITU-T Recommendation Y.2011, ITU-T, 2004.

[9] Aleg Cherp, "Greenhouse Gas Accounting Standards: Applications and Approaches", 2003.

[10] Soraya Kouadri Most'efaoui, B'eat Hirsbrunner: Context Aware Service Provision, Proceedings of the IEEE/ACS International Conference on Pervasive Services (ICPS'04), IEEE Computer Society (2004)

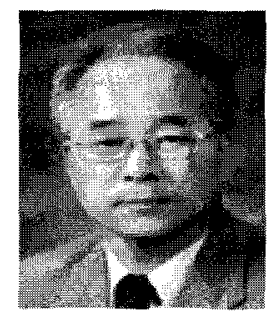

Sam-gil Choi Received the Ph.D degree in electronics engineering from Dong A University, Korea, in 2001. Since 1985, he has been at Dong-Eui University, Korea, where he is a professor at the information and communication engineering department. From 2005 to 2006 , he was Dean college of Engineering Dong-Eui University. And also, He was a visiting professor at CSU Sacramento in USA. His research interests are signal analysis of communication networks, protocols in wireless networks of ICT

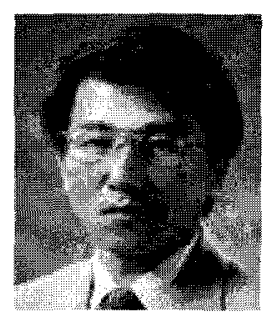

Dong-il Kim Received the Ph.D degree in electronics information and communication engineering from Kwang-woon University, Korea, in 1992. Since 1991, he has been at Dong-Eui University, Korea, where he is a professor at the information and communication engineering department. From 1983 to 1991 , he was a general manager at switching research center in $L G$ information and communication institute. He was a visiting researcher at standardization research center, ETRI from 1998 to 1999. He is Editor journal of KICS. He was designated as an IT Standard Expert on behalf of Korea by the KCC in 2002. He is an Editor in the ITUT SG13 and a Member of KIMICS. His research interests are analysis of performance in a communication networks, protocols in wireless networks and standardization of ICT

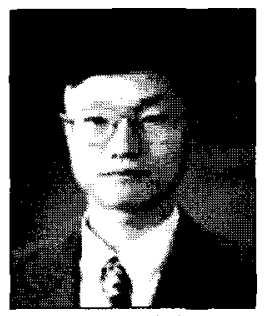

Soong- Hee Lee Received the B.S., M.S., and Ph.D. degrees from Kyungpook National University, Daegu in 1987, 1990, and 1995 , respectively. From 1987 to 1997 , he was a member of research staff in Electronics and Telecommunications Research Institute. Since 1997, he has been with the Dep't of Information and Communications Engineering, Inje University as an associate professor. His research activities are in the area of next generation network technologies and services. He was designated as an IT Standard Expert on behalf of Korea by the Ministry of Information and Communications in 2001. He is an Editor in the ITU-T SG13 and a Member of KIMICS. 